D

U251 read count for different regions

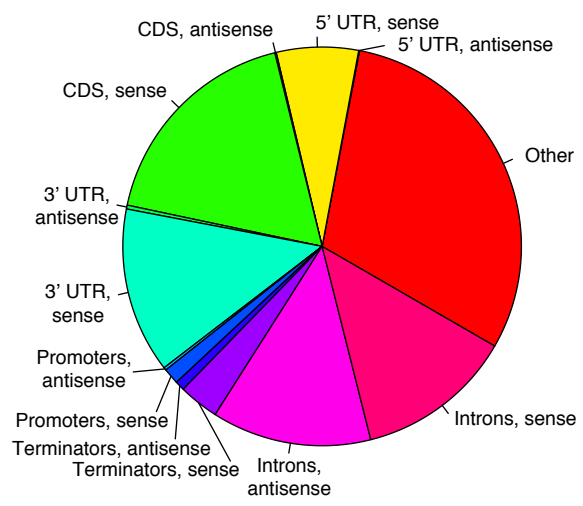

$\mathrm{G}$ U2-OS read count for different regions

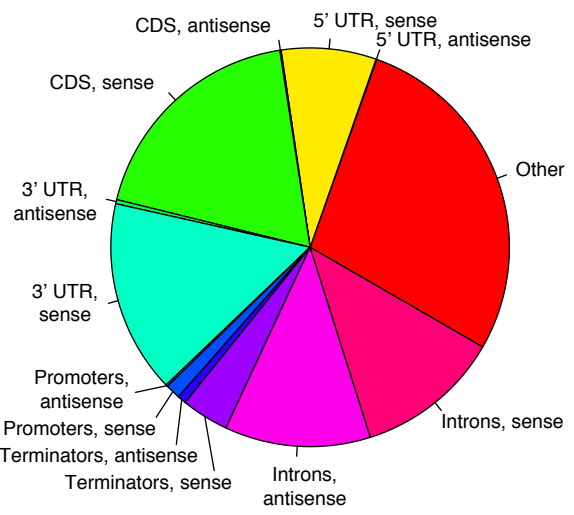

E

Relative expression desities

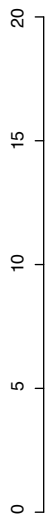

$\sqrt{10}$

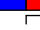

Genome

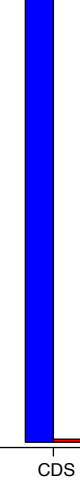

F

\section{- Sense}

Antisense

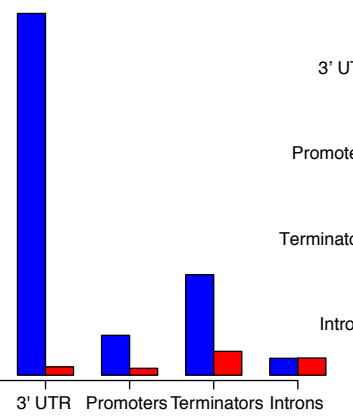

$\mathrm{H}$

Relative expression desities

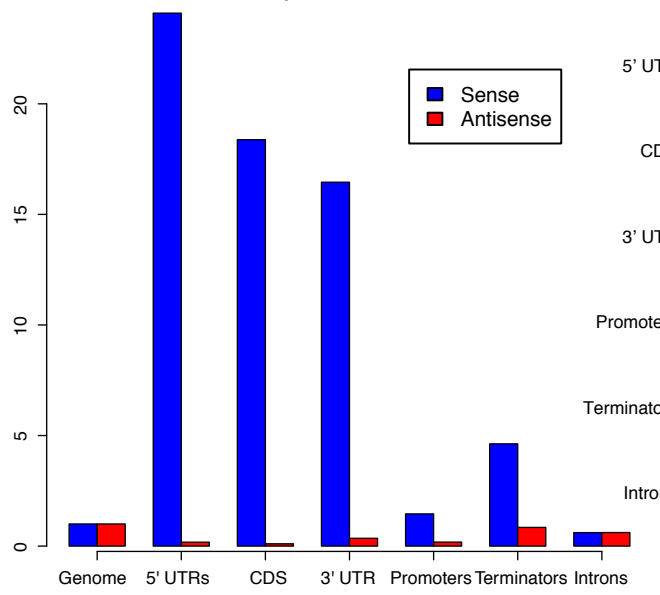

$C$

, UTR

CDS

UTR
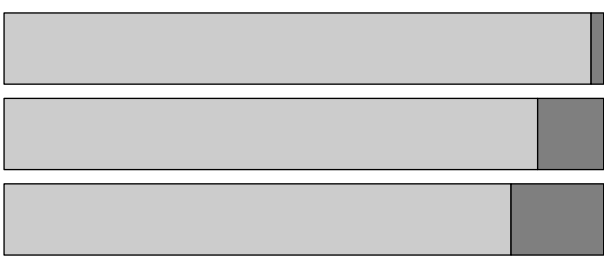

trons

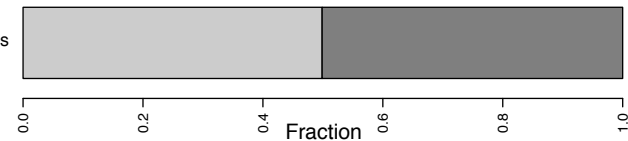

Fraction of reads that match the sense and antisense strands and antisense strands

UTR

CDS
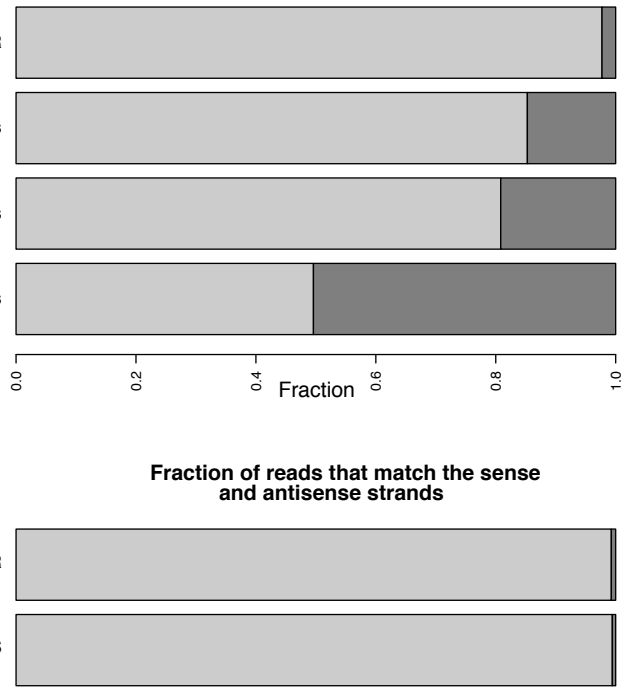